\title{
Diastereoselective preparation of cyclic $\beta$-aminoketals. Application to the total synthesis of piperidine alkaloids
}

\author{
Ala Eddin Al-Sarabi, Annabelle Bariau, Marion Gabant, Jean-Charles Wypych, \\ Pierre Chalard, and Yves Troin*
}

Laboratoire de Chimie des Hétérocycles et des Glucides, EA 987, Ecole Nationale Supérieure de Chimie de Clermont-Ferrand, Université Blaise Pascal, BP 187, 63174 Aubière Cedex, France

E-mail:troin@chimie.univ-bpclermont.fr

\section{Dedicated to Professor Jean Claude Gramain on the occasion of his $\mathbf{7 0}^{\text {th }}$ birthday}

\begin{abstract}
We describe here the diastereoselective synthesis of cyclic $\beta$-aminoketone derivatives which are very powerful synthons for alkaloid synthesis. They were stereoselectively prepared by condensation of a chiral lithium amide on an $\alpha, \beta$-unsaturated ester which possesses a leaving group in $\omega$-position. The usefulness of these compounds was demonstrated through the synthesis of indolizidine 209D, an alkaloid isolated from skin extracts of South American frogs and also in the preparation of allosedridine which has been isolated from Sedum acre.
\end{abstract}

Keywords: Diastereoselective synthesis, Mannich Reaction, alkaloids, indolizidine 209D, allosedridine

\section{Introduction}

The piperidine moiety constitutes a structural unit of many alkaloids presenting various biological activities. ${ }^{1}$ For this main reason, stereoselective synthesis of this framework is the object of continuous and intensive efforts. ${ }^{2}$ Among them, our laboratory has developed an original approach which relies on the use of an aldehyde and an $\alpha$-chiral $\beta$-aminoketal, together involved in a Mannich type cyclization, to give rapidly and highly stereoselective access to the corresponding cis-2,6-disubstituted piperidines (Scheme 1). This methodology has been successfully applied to the total synthesis of various alkaloids ${ }^{3}$. 


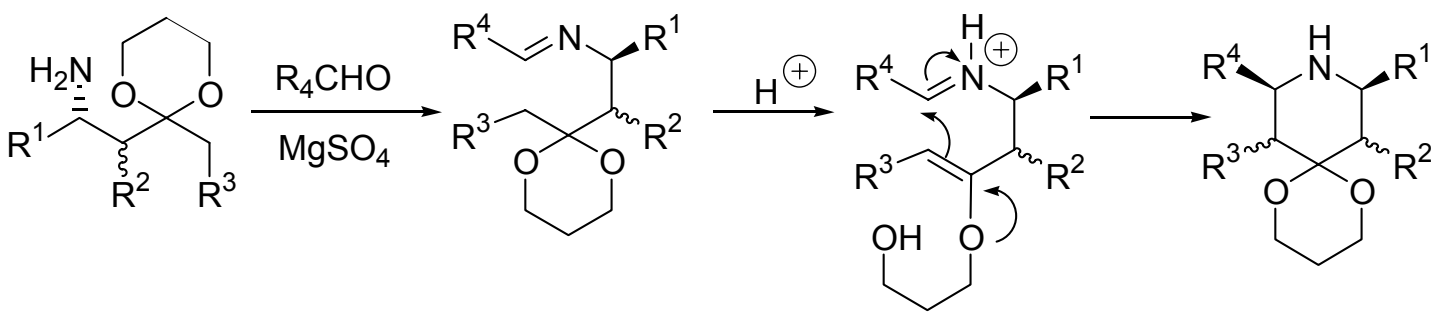

\section{Scheme 1}

In order to explore the scope and limitation of this strategy, we decided to try, as new precursor, a "preformed" cyclic $\beta$-aminoketal, compounds $\mathbf{1}$ or $\mathbf{2}$, in the cyclization step. Accordingly, we reasoned that this strategy, applied to aldehyde derivatives, should furnish a rapid stereoselective entry to a bicyclic ring system 4 via intramolecular cyclization of the intermediate 3 (Scheme 2). Moreover, if we were able to prepare efficiently the cyclic $\beta$ aminoketal $\mathbf{1}$ or $\mathbf{2}$ in a non racemic form, we could hope that a chiral bicyclic compound could be obtained.
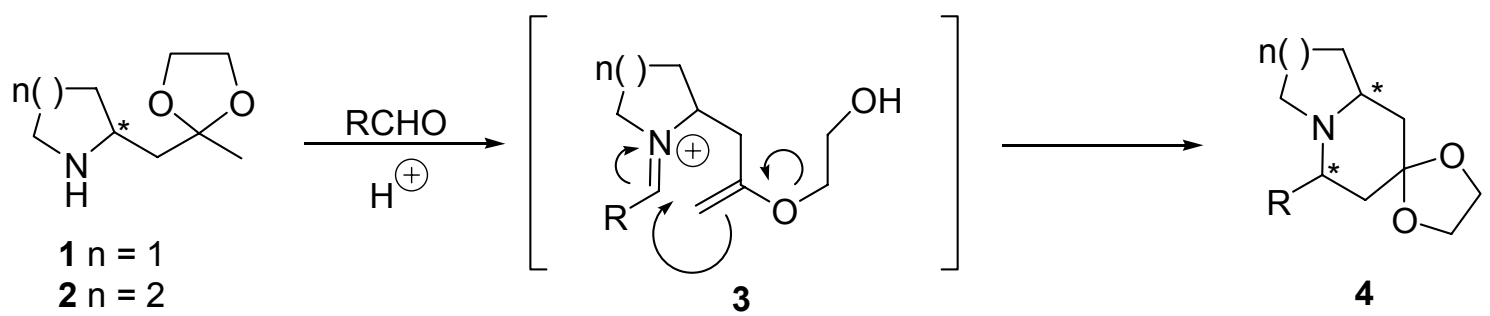

\section{Scheme 2}

Cyclic $\beta$-aminoketal derivatives $\mathbf{1}$ and $\mathbf{2}$ were prepared according to our general method previously described ${ }^{4}$. The key step of this synthesis involves a highly diastereoselective addition of a chiral lithium amide on an $\alpha, \beta$-unsaturated ester ${ }^{5}$ which could be further transformed into a ketone. When the ester has a potential leaving group (LG) in $\omega$-position, intramolecular

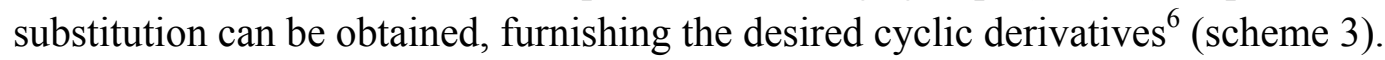




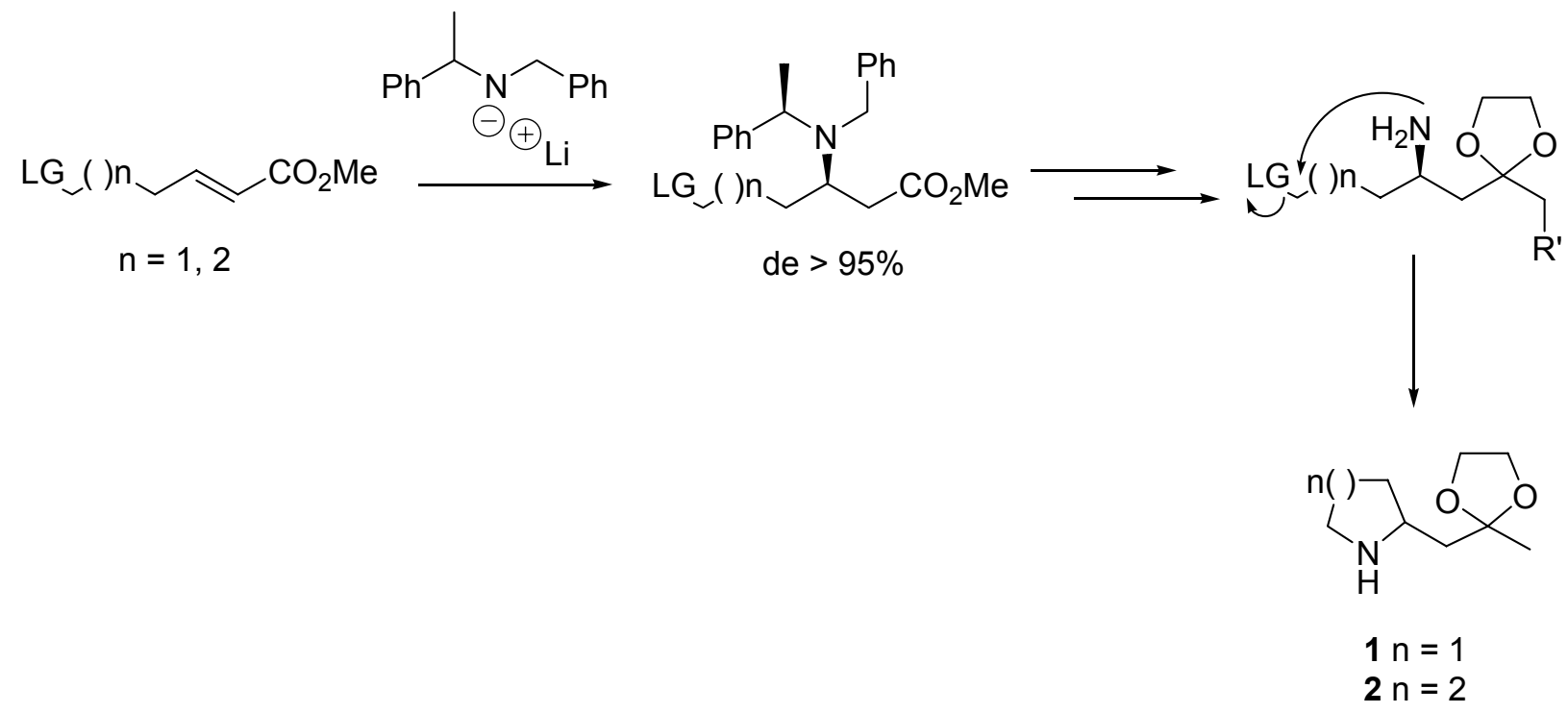

Scheme 3

\section{Results and Discussion}

The preparation of the cyclic $\beta$-aminoketals was realized as described in Scheme 4 . The $\omega$ functionalized $\alpha, \beta$-unsaturated esters $5^{6,7}$ and $\mathbf{6}^{6,7}$ were prepared in two steps starting from the corresponding commercial $\alpha, \omega$-chloroalcohols, by oxidation of the alcohol function in an aldehyde followed by a Wittig-Horner reaction with carbomethoxymethyltriphenylphosphorane.

The condensation of the chiral amide $\mathbf{7}$ on esters $\mathbf{5}$ and $\mathbf{6}$ provided the Michael adducts $\mathbf{8}$ and 9 in good yields and excellent diastereoselectivity (de $>95 \%$ ). The selective cleavage of the para-methoxybenzyl group was realized using ceric ammonium chloride which led to compounds $\mathbf{1 0}$ and $\mathbf{1 1}$ in a very fast and clean reaction. Cyclization of compound $\mathbf{1 0}$ was first realized in the presence of potassium iodide and potassium carbonate in refluxing ethanol. ${ }^{8}$ An equimolecular mixture of two diastereoisomers 12 and $\mathbf{1 3}$ (epimers at C-2 position), was obtained with an average yield of 58\% (Scheme 5). The relative configuration of these two diastereosiomers has been established by comparison of their NMR data with those described in the literature. ${ }^{9}$ This epimerization process, already observed in alkaloid synthesis ${ }^{10}$, could be explained by a retro-Michael type reaction occurring on cyclized compound $\mathbf{1 2}$, consecutively to the extended reflux in basic conditions. When the reaction was carried out under milder conditions (methanol, room temperature), the cyclic $\beta$-aminoesters 12 and $\mathbf{1 4}$ were now obtained stereoselectively and with better yields. Treatment of the ester with $\mathrm{N}, \mathrm{O}$-dimethylhydroxylamine hydrochloride in dichloromethane at $0^{\circ} \mathrm{C}$ in the presence of trimethylaluminium led to the corresponding Weinreb amide 15 and 16, ${ }^{11}$ precursors of the corresponding ketones. These two synthons were then separately involved in total alkaloid synthesis. 


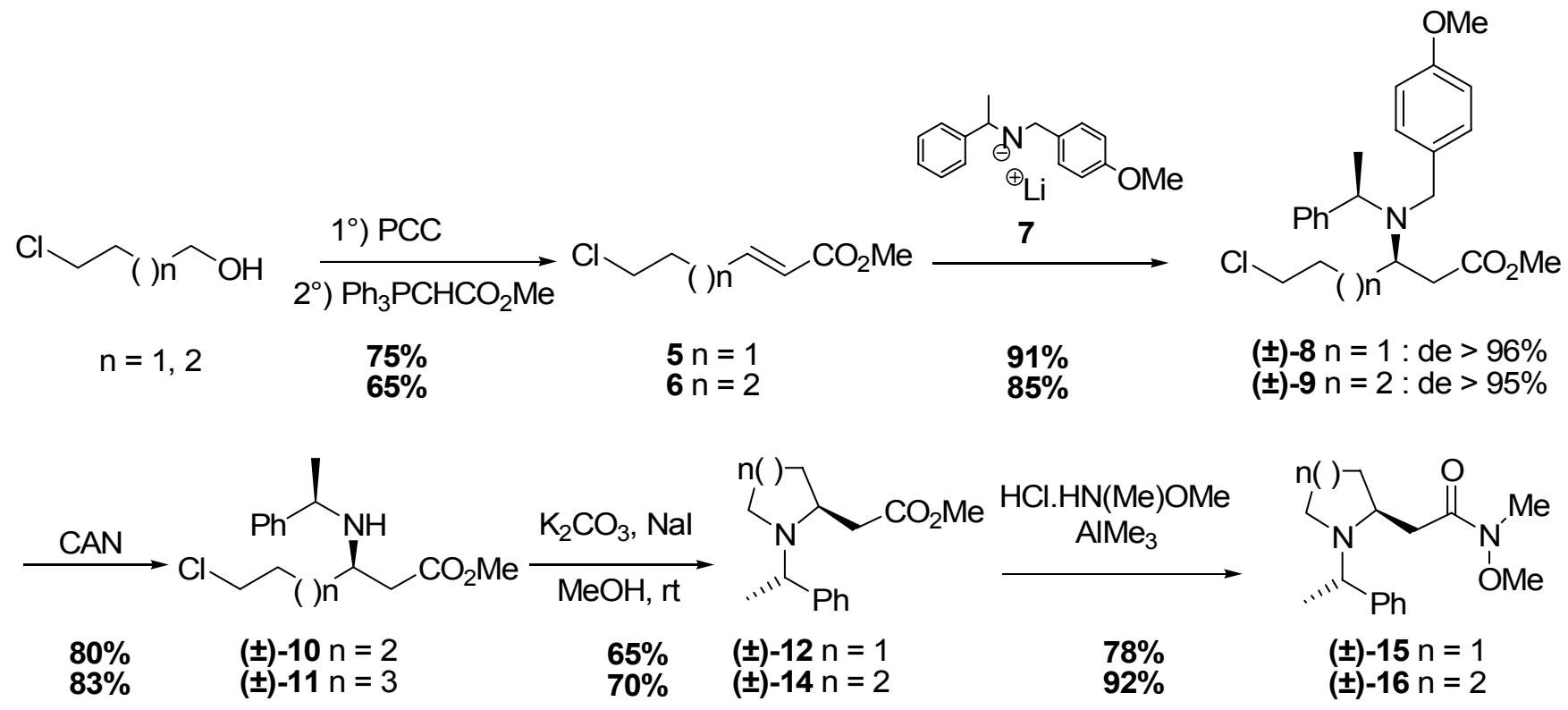

\section{Scheme 4}<smiles>CCOC(=O)CC(CCCl)N[C@@H](CC(=O)OCC)c1ccccc1</smiles>

(士)-10

$58 \%$

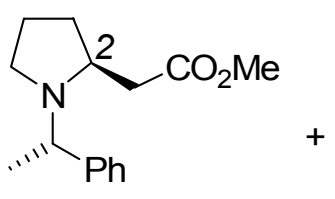

(士)-12<smiles>CC(=O)C[C@H]1CCCN1[C@@H](C)c1ccccc1</smiles>

(士)-13

$50 / 50$

\section{Scheme 5}

Amide 15 was used for the preparation of indolizidine 209D. ${ }^{12}$ This alkaloid was isolated from cutaneous glandular secretions of the South American batrachians of the Dendrobates species. As others compounds of the same family, this indolizidine acted as noncompetitive inhibitor of the nicotinic receptor and thus has an activity on the central nervous system. ${ }^{13}$ Thus, amide 15 was first transformed into ketone 17 using methylmagnesium bromide. Protection of the carbonyl group under mild conditions ${ }^{14}$ (trimethylorthoformate, $\mathrm{p}-\mathrm{TsOH}$, ethylene glycol, room temperature) led to the $\beta$-aminoketal 18 in $74 \%$ yield (Scheme 6). 


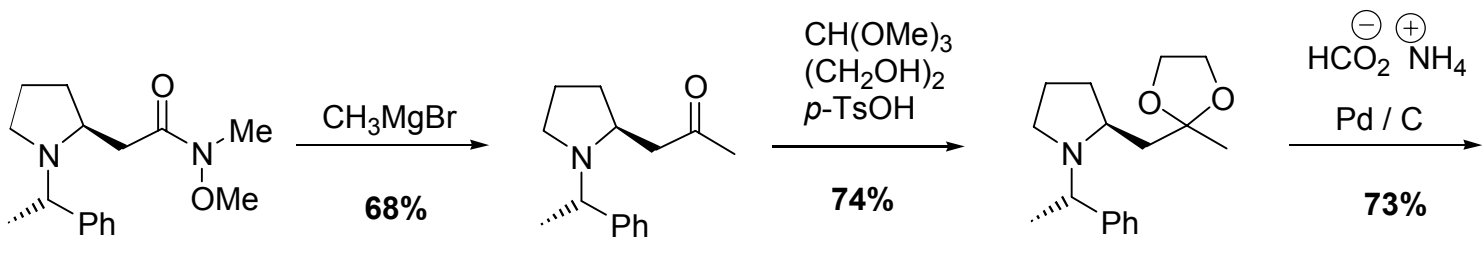

(士)-15

(士)-17

(士)-18

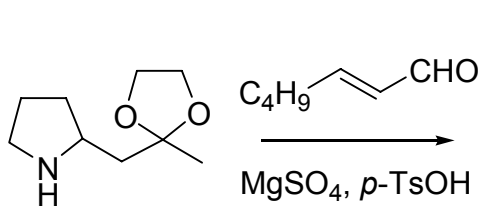

$( \pm)-1$
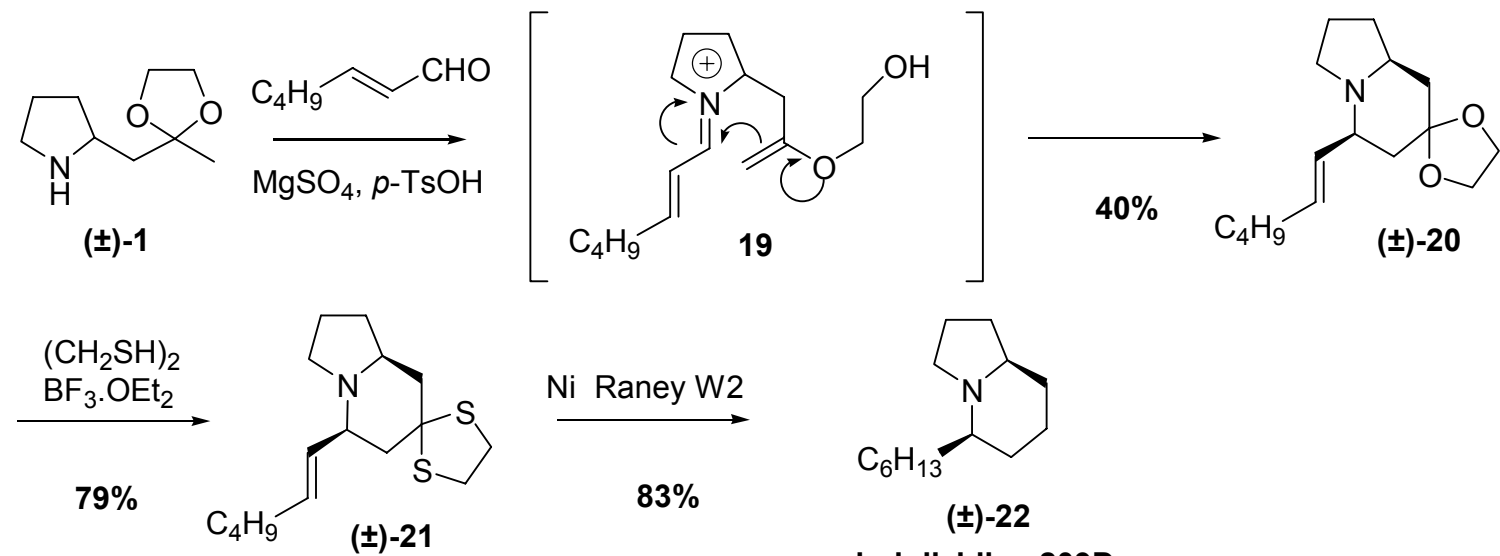

Ni Raney W2

$83 \%$

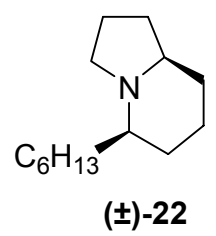

indolizidine 209D

\section{Scheme 6}

Treatment of amine $\mathbf{1 8}$ with ammonium formate in the presence of palladium on charcoal in refluxing methanol led to the desired cyclic aminoketal $\mathbf{1}$ in $73 \%$ yield. Condensation of amine $\mathbf{1}$ with hepten-2-al followed by acidic treatment led in a stereoselective manner to the indolizidine 20 in $40 \%$ yield. Transacetalation of compound $\mathbf{2 0}$ was carried out using ethanedithiol in dichloromethane in the presence of borontrifluoride etherate complex and gave $\mathbf{2 1}$ in $79 \%$ yield. ${ }^{15}$ Further hydrogenolysis of thioketal $\mathbf{2 1}$ in refluxing methanol (W2 Raney nickel ${ }^{16}$ ) furnished $( \pm$ )-indolizidine 209D 22 with an excellent yield. The spectroscopic data obtained for this compound are in full agreement with those described in the literature. ${ }^{12}$.

On the other hand, piperidine derivative $\mathbf{1 6}$ is an excellent precursor for the stereoselective synthesis of $( \pm)$-allosedridine, ${ }^{17}$ a piperidine alkaloid isolated from Sedum Acre. ${ }^{18}$ Treatment of amide 16 with methylmagnesium bromide (Scheme 7) led to ketone 23, albeit in moderate yield, mainly due to problems of stability and purification. To circumvent this problem, we decided to change the nitrogen protective group. Hydrogenolysis of the $\alpha$-methylbenzyl group of 16 with palladium on charcoal in the presence of ammonium formate furnished piperidine $\mathbf{2 4}$, which, without further purification, was immediately reprotected with benzyl chloroformate. ${ }^{19}$ By this route, piperidine $\mathbf{2 5}$ was obtained in two steps with a 70\% overall yield after purification. 
<smiles>CON(C)C(=O)C[C@@H]1CCCCN1[C@H](C)c1ccccc1</smiles>

(士)-16<smiles>CON(C)C(=O)CC1CCCCC1CC(N)=O</smiles><smiles>CON(C(=O)O)C(=O)CC1CCCCN1C(=O)OCc1ccccc1</smiles>

not isolated<smiles>CC(=O)CC1CCCCN1C(=O)OCc1ccccc1</smiles>

$( \pm)-26$

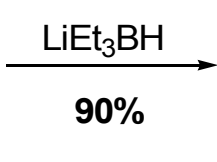

$\mathrm{H}_{2} / \mathrm{Pd}(\mathrm{OH})_{2} / \mathrm{C}$<smiles>CC(C)[C@@H](O)C[C@@H]1CCCCN1C(=O)OC(C)(C)C</smiles>

$( \pm)-27$

\section{Scheme 7}

Amide 25 was now treated with methylmagnesium bromide and provided ketone 26. Reduction of the latter using Superhydride ${ }^{\circledR}$ reagent furnished, with a good yield, two diastereoisomeric alcohols 27 and 28 (7/3 ratio) which were easily separated by silica gel chromatography. The relative configuration of the stereogenic center of each compound was established by comparison of their spectroscopic data with those published in the literature ${ }^{17 \mathrm{c}}$. Finally, hydrogenolysis of the carbamate group in the presence of Pearlman's catalyst led to $( \pm)$ allosedridine $\mathbf{2 9}$ with a very good yield. 


\section{Conclusions}

In conclusion, we have described the stereoselective synthesis of cyclic $\beta$-aminoketals. As their acyclic corresponding compounds, they could be used for intramolecular Mannich type reaction. Using this methodology, total syntheses of $( \pm)$-indolizidine 209D and $( \pm)$-allosedridine were achieved in respectively 10 and 7 steps from commercial starting materials. This synthetic scheme can now be applied to the preparation of more complex alkaloids in non-racemic series. This work is currently under progress and will be reported in due course.

\section{Acknowledgments}

We thank the Ministère de la Jeunesse, de l'Education Nationale et de la Recherche for financial support.

\section{Experimental Section}

General Procedures. Unless otherwise specified, reagents were obtained from commercial suppliers. Solvents were dried and freshly distilled following the usual procedures. Product organic solutions were dried over sodium sulfate prior to evaporation of the solvents under reduced pressure on a rotatory evaporator. Thin layer chromatography was performed on TLC precoated aluminium backed silica plates and spots were visualized using UV light (254 nm) before using ethanolic phosphomolybdic acid solution (heating). Column chromatography was carried out on silica gel (70-230 mesh). ${ }^{1} \mathrm{H}$ and ${ }^{13} \mathrm{C}$ NMR spectra were measured at 400.13 and 100.61 MHz respectively. Chemicals shifts are reported in ppm relative to $\mathrm{SiMe}_{4}$. Signals are quoted s (singlet), d (doublet), $\mathrm{t}$ (triplet), q (quartet), m (multiplet), br (broad) and coupling constant (J) values are given in Hz. Infrared spectra were recorded on a FTIR spectrophotometer. Compositions of stereoisomeric mixtures were determined by NMR analysis on crude products before any purification. High Resolution Electro Spray Impact Mass Spectra (HR-ESI-MS) were obtained from the Centre Régional de Mesures Physiques de l'Université Blaise Pascal (Clermont II), France.

\section{General procedure for preparation of $\beta$-aminoesters 8 and 9}

To a cold $\left(0^{\circ} \mathrm{C}\right)$ solution of $N$-para-methoxybenzyl- $N$ - $\alpha$-methyl benzylamine 7 (1.1 equiv) in dry THF ( $5 \mathrm{~mL} / \mathrm{mmol}$ of 7) was added slowly under argon n-butyllithium solution $1.6 \mathrm{M}$ in hexanes (1.2 equiv.). The resultant pink solution of lithium amide was stirred for 15 minutes then cooled at $-78^{\circ} \mathrm{C}$ before dropwise addition of a solution of $\alpha, \beta$-unsaturated ester $(\mathbf{5}$ or $\mathbf{6})$ (1 equiv.) in dry THF $(2 \mathrm{~mL} / \mathrm{mmol}$ of 5 or $\mathbf{6})$. The mixture was stirred at $-78^{\circ} \mathrm{C}$ until TLC showed no starting conjugated ester. Then a saturated $\mathrm{NH}_{4} \mathrm{Cl}$ aqueous solution was added dropwise and the resulting 
solution was allowed to warm to room temperature. $\beta$-aminoester (8 or 9$)$ was then extracted with diethylether. Combined organic extracts were dried, filtered and evaporated. The crude product was purified by column chromatography.

Methyl (3R*)-6-chloro-3-[(N-4-methoxybenzyl)-( $N-\left(1 R^{*}\right) 1-$ phenylethyl)]amino)-hexanoate (8). Following the general procedure for the preparation of $\beta$-aminoester, ester 5 (12.3 mmol, 2.0 g) and amine $7(5.6 \mathrm{~mL})$ afforded, after purification on silica gel (ethyl acetate:cyclohexane 1:3), $\beta$-aminoester 8 as a yellow oil (4.5 g, 91\%): $d e>96 \%$ (NMR data); IR (film, $\mathrm{cm}^{-1}$ ) 1724, 1610, 1510, 1436, 1250; ${ }^{1} \mathrm{H}$ NMR (400 MHz, $\left.\mathrm{CDCl}_{3}\right) \delta 7.10(7 \mathrm{H}, \mathrm{m}), 6.75(2 \mathrm{H}, \mathrm{d}, \mathrm{J}=8.0 \mathrm{~Hz}), 3.70$ $(1 \mathrm{H}, \mathrm{q}, \mathrm{J}=7.0 \mathrm{~Hz}), 3.65(3 \mathrm{H}, \mathrm{s}), 3.60-3.50(2 \mathrm{H}, \mathrm{m}), 3.40(3 \mathrm{H}, \mathrm{s}), 3.30(2 \mathrm{H}, \mathrm{m}), 3.15(1 \mathrm{H}, \mathrm{m})$, $1.90-1.40(6 \mathrm{H}, \mathrm{m}), 1.20(3 \mathrm{H}, \mathrm{d}, \mathrm{J}=7.0 \mathrm{~Hz}) ;{ }^{13} \mathrm{C} \mathrm{NMR}\left(100 \mathrm{MHz}, \mathrm{CDCl}_{3}\right): \delta 173.1,158.5,142.8$, 133.0, 129.3, 128.2, 127.8, 127.1, 113.7, 57.3, 55.3, 53.1, 51.5, 49.2, 45.1, 36.3, 30.7, 30.2, 19.6.

Methyl (3R*)-7-chloro-3-[( $N$-4-methoxybenzyl)-( $N-\left(1 R^{*}\right) 1-$ phenylethyl $\left.)\right]$ amino $)$-heptanoate (9). Following the general procedure for the preparation of $\beta$-aminoester, ester 6 ( $2.83 \mathrm{mmol}, 0.5$ g) and amine $7(1.3 \mathrm{~mL})$ afforded, after purification on silica gel (ethyl acetate:cyclohexane 1:3), $\beta$-aminoester 9 as a yellow oil (1.0 g, 85\%): $d e=95 \%$ (NMR data); IR (film, $\mathrm{cm}^{-1}$ ) 1725, 1615, 1508, 1445, 1256; ${ }^{1} \mathrm{H}$ NMR (400 MHz, $\left.\mathrm{CDCl}_{3}\right) \delta 7.30(7 \mathrm{H}, \mathrm{m}), 6.90(2 \mathrm{H}, \mathrm{d}, \mathrm{J}=8.0 \mathrm{~Hz}), 3.90$ $(1 \mathrm{H}, \mathrm{q}, \mathrm{J}=7.0 \mathrm{~Hz}), 3.80(3 \mathrm{H}, \mathrm{s}), 3.60\left[2 \mathrm{H}, \mathrm{AB}\right.$ system $\Delta v=80.0 \mathrm{~Hz}, \delta_{\mathrm{A}}=3.70\left(1 \mathrm{H}, \mathrm{d}, \mathrm{J}_{\mathrm{AB}}=\right.$ $\left.15.0 \mathrm{~Hz}), \delta_{\mathrm{B}}=3.50\left(1 \mathrm{H}, \mathrm{d}, \mathrm{J}_{\mathrm{AB}}=15.0 \mathrm{~Hz}\right)\right], 3.55(3 \mathrm{H}, \mathrm{s}), 3.52(2 \mathrm{H}, \mathrm{m}), 3.25(1 \mathrm{H}, \mathrm{m}), 2.00(2 \mathrm{H}$, m), $1.80-1.40(4 \mathrm{H}, \mathrm{m}), 1.35(3 \mathrm{H}, \mathrm{d}, \mathrm{J}=7.0 \mathrm{~Hz}), 1.25(2 \mathrm{H}, \mathrm{m}) ;{ }^{13} \mathrm{C}$ NMR $\left(100 \mathrm{MHz}, \mathrm{CDCl}_{3}\right): \delta$ 173.2 , 158.5, 143.0, 133.3, 129.3, 128.1, 127.9, 127.0, 113.7, 57.5, 55.3, 53.5, 51.4, 49.1, 45.1, 36.4, 32.7, 32.4, 24.3, 14.2; HRMS Calcd. $\mathrm{C}_{24} \mathrm{H}_{32} \mathrm{NO}_{3} \mathrm{Cl}\left[\mathrm{M}^{+}\right]$: 417.2070. Found: 417.2051.

\section{General procedure for preparation of mono protected amines 10 and 11}

To a cold $\left(0^{\circ} \mathrm{C}\right)$ solution of $\beta$-aminoester (8 or 9) (1 equiv) in a 1:1 mixture of water and acetonitrile ( $3 \mathrm{~mL} / \mathrm{mmol}$ of $\beta$-aminoester) CAN (3.0 equiv.) was added. The resultant solution was stirred for 30 minutes then hydrolized with a saturated aqueous $\mathrm{NaCl}$ solution. The resulting mono protected amine (10 or 11) was then extracted with ethyl acetate and the combined organic extracts were dried over sodium sulfate, filtered and evaporated. The crude product was purified by column chromatography.

Methyl (3R*)-6-chloro-3-[N-(1R*)1-phenylethyl)]amino)-hexanoate (10). Following the general procedure for the preparation of mono protected amine, $\beta$-aminoester 8 (3.72 mmol, 1.5 g) and CAN $(6.11 \mathrm{~g})$ afforded, after purification on silica gel (ethyl acetate:cyclohexane 1:5 then ethyl acetate:methanol 9:1), amine 10 as a yellow oil $\left(0.85 \mathrm{~g}, 80 \%\right.$ ): IR (film, $\mathrm{cm}^{-1}$ ) 3512, 2956, 1735, 1610, 1510, 1436, 1250; ${ }^{1} \mathrm{H}$ NMR (400 MHz, $\left.\mathrm{CDCl}_{3}\right) \delta 7.60(2 \mathrm{H}, \mathrm{d}, \mathrm{J}=8.0 \mathrm{~Hz}), 7.45$ $(3 \mathrm{H}, \mathrm{m}), 4.50(1 \mathrm{H}, \mathrm{m}), 3.70(3 \mathrm{H}, \mathrm{s}), 3.35(3 \mathrm{H}, \mathrm{m}), 3.05(1 \mathrm{H}, \mathrm{dd}, \mathrm{J}=17.5$ and $6.0 \mathrm{~Hz}), 3.75(1 \mathrm{H}$, $\mathrm{dd}, \mathrm{J}=17.5$ and $5.5 \mathrm{~Hz}), 2.50(1 \mathrm{H}, \mathrm{bs}), 1.90(2 \mathrm{H}, \mathrm{m}), 1.80(3 \mathrm{H}, \mathrm{d}, \mathrm{J}=6.5 \mathrm{~Hz}), 1.70(\mathrm{~m}, 2 \mathrm{H}) ;{ }^{13} \mathrm{C}$ NMR (100 MHz, $\left.\mathrm{CDCl}_{3}\right): \delta 170.8,135.7,129.8,129.6,127.8,58.1,53.2,52.5,43.8,35.1,29.6$, 27.9, 20.1; HRMS Calcd. $\mathrm{C}_{15} \mathrm{H}_{22} \mathrm{NO}_{2} \mathrm{Cl}\left[\mathrm{M}^{+}\right]-\mathrm{CH}_{3}$ : 268.1104. Found: 268.1130.

Methyl (3R*)-7-chloro-3-[N-(1R*)1-phenylethyl)]amino)-heptanoate (11). Following the general procedure for the preparation of mono protected amine, $\beta$-aminoester 9 (2.5 mmol, 1.04 
g) and CAN (4.11 g) afforded, after purification on silica gel (ethyl acetate:cyclohexane 1:5 then ethyl acetate:methanol 9:1), amine $\mathbf{1 0}$ as a yellow oil (0.62 g, 83\%): IR (film, $\mathrm{cm}^{-1}$ ) 3480, 2958, 1736, 1612, 1524, 1460, 1244; ${ }^{1} \mathrm{H}$ NMR (400 MHz, $\left.\mathrm{CDCl}_{3}\right) \delta$ 7.55-7.35 (5H, m), $4.50(1 \mathrm{H}, \mathrm{q}, \mathrm{J}$ $=7.0 \mathrm{~Hz}), 4.35(1 \mathrm{H}, \mathrm{bs}), 3.70(3 \mathrm{H}, \mathrm{s}), 3.40(2 \mathrm{H}, \mathrm{dt}, \mathrm{J}=6.5$ and $2 \mathrm{~Hz}), 3.30(1 \mathrm{H}, \mathrm{m}), 3.00(1 \mathrm{H}$, $\mathrm{dd}, \mathrm{J}=17.5$ and $6.0 \mathrm{~Hz}), 2.70(1 \mathrm{H}, \mathrm{dd}, \mathrm{J}=17.5$ and $6.5 \mathrm{~Hz}), 1.85(3 \mathrm{H}, \mathrm{d}, \mathrm{J}=7.0 \mathrm{~Hz}), 1.80-1.50$ $(4 \mathrm{H}, \mathrm{m}), 1.45(2 \mathrm{H}, \mathrm{m}) ;{ }^{13} \mathrm{C} \mathrm{NMR}\left(100 \mathrm{MHz}, \mathrm{CDCl}_{3}\right): \delta 171.0,135.7,129.8,129.6,127.6,58.2$, 53.7, 52.4, 44.2, 35.0, 31.5, 31.2, 22.4, 20.1; HRMS Calcd. $\mathrm{C}_{16} \mathrm{H}_{24} \mathrm{NO}_{2} \mathrm{Cl}\left[\mathrm{M}^{+}\right]-\mathrm{CH}_{3}: 282.1261$. Found: 282.1260 .

\section{Cyclization of amine 10 in refluxing ethanol}

To a solution of amine 10 (1 equiv., $3.00 \mathrm{~g}, 10.6 \mathrm{mmol})$ in ethanol $(70 \mathrm{~mL})$ was added potassium carbonate (1.1 equiv., $1.60 \mathrm{~g}, 11.6 \mathrm{mmol}$ ), then sodium iodide (1.1 equiv., $1.74 \mathrm{~g}, 11.6 \mathrm{mmol}$ ). The resulting solution was stirred 1 hour under reflux before the solvent was removed in vacuo. The crude mixture was dissolved in diethyl ether and hydrolysed with a saturated aqueous $\mathrm{NaCl}$ solution. The combined organic layers were dried over sodium sulfate, filtered and evaporated. The crude product was purified by column chromatography to afford a $1 / 1$ mixture of the two pyrrolidines diastereoisomers 12 and $13(1.5 \mathrm{~g})$ in 58\% overall yield, as a pale yellow oil.

Mixture of $\left(R^{*}, R^{*}\right)$ and $\left(R^{*}, S^{*}\right)$ Methyl [1-(1-phenylethyl)pyrrolidin-2-yl]acetate12 and 13. ${ }^{1} \mathrm{H}$ NMR (400 MHz, $\left.\mathrm{CDCl}_{3}\right) \delta$ 7.40-7.20 (5H, m), $3.75(0.5 \mathrm{H}, \mathrm{q}, \mathrm{J}=7.0 \mathrm{~Hz}), 3.70(0.5 \mathrm{H}, \mathrm{q}, \mathrm{J}$ $=6.5 \mathrm{~Hz}), 3.65(1.5 \mathrm{H}, \mathrm{s}), 3.60(1.5 \mathrm{H}, \mathrm{s}), 3.30(0.5 \mathrm{H}, \mathrm{m}), 3.15(0.5 \mathrm{H}, \mathrm{m}), 2.75(1 \mathrm{H}, \mathrm{m}), 2.60$ $(0.5 \mathrm{H}, \mathrm{dd}, \mathrm{J}=14.5$ and $3.5 \mathrm{~Hz}), 2.55-2.30(1.5 \mathrm{H}, \mathrm{m}), 2.28(0.5 \mathrm{H}, \mathrm{dd}, \mathrm{J}=14.5$ and $9.5 \mathrm{~Hz}), 2.15$ $(0.5 \mathrm{H}, \mathrm{dd}, \mathrm{J}=15.0$ and $8.0 \mathrm{~Hz}), 1.90-1.80(1 \mathrm{H}, \mathrm{m}), 1.75-1.50(3 \mathrm{H}, \mathrm{m}), 1.45(1.5 \mathrm{H}, \mathrm{d}, \mathrm{J}=6.5 \mathrm{~Hz})$, $1.38(1.5 \mathrm{H}, \mathrm{d}, \mathrm{J}=7.0 \mathrm{~Hz}) ;{ }^{13} \mathrm{C} \mathrm{NMR}\left(100 \mathrm{MHz}, \mathrm{CDCl}_{3}\right): \delta 173.0,172.9,145.1,142.0,129.6$, $128.8,128.3,128.1,127.2,127.8,60.7,60.5,58.0,56.8,51.4,51.3,50.1,50.0,40.0,39.3,30.9$, $30.8,22.7,22.1,20.8,18.4$.

\section{General procedure for the cyclization of mono protected amines}

To a solution of mono protected amine (10 or 11) (1 equiv) in methanol (7 mL/mmol of mono protected amine) was added potassium carbonate (1.1 equiv.) then sodium iodide (1.1 equiv.). The resulting solution was stirred 2 hours at room temperature before the solvent was removed in vacuo. The crude mixture was dissolved in diethyl ether and hydrolysed with a saturated aqueous $\mathrm{NaCl}$ solution. The combined organic layers were dried over sodium sulfate, filtered and evaporated. The crude product was purified by column chromatography.

$\left(R^{*}, R^{*}\right)$ - Methyl [1-(1-phenylethyl)pyrrolidin-2-yl]acetate (12). Following the general procedure for cyclization of mono protected amine, amine 10 (11.3 mmol, $3.20 \mathrm{~g}), \mathrm{K}_{2} \mathrm{CO}_{3}(1.75$ g) and $\mathrm{NaI}(1.90 \mathrm{~g})$ afforded, after purification on silica gel (ethyl acetate:cyclohexane 1:3), pyrrolidine 12 as a yellow oil (1.81 g, 65\%): IR (film, $\mathrm{cm}^{-1}$ ) 2986, 1726, 1615, 1513, 1438, 1258; ${ }^{1} \mathrm{H}$ NMR (400 MHz, $\left.\mathrm{CDCl}_{3}\right) \delta 7.35(5 \mathrm{H}, \mathrm{m}), 3.70(1 \mathrm{H}, \mathrm{q}, \mathrm{J}=6.5 \mathrm{~Hz}), 3.60(3 \mathrm{H}, \mathrm{s}), 3.10(1 \mathrm{H}$, $\mathrm{m}), 2.75(1 \mathrm{H}, \mathrm{ddd}, \mathrm{J}=9.0,6.5$ and $2.5 \mathrm{~Hz}), 2.60(1 \mathrm{H}, \mathrm{dd}, \mathrm{J}=14.5$ and $3.5 \mathrm{~Hz}), 2.40(1 \mathrm{H}, \mathrm{dt}, \mathrm{J}=$ 9.0 and $7.0 \mathrm{~Hz}), 2.30(1 \mathrm{H}, \mathrm{dd}, \mathrm{J}=14.5$ and $9.5 \mathrm{~Hz}), 1.85(1 \mathrm{H}, \mathrm{m}), 1.70(1 \mathrm{H}, \mathrm{m}), 1.60(2 \mathrm{H}, \mathrm{m})$, $1.40(3 \mathrm{H}, \mathrm{d}, \mathrm{J}=6.5 \mathrm{~Hz}) ;{ }^{13} \mathrm{C}$ NMR $\left(100 \mathrm{MHz}, \mathrm{CDCl}_{3}\right): \delta 172.9,142.0,129.6,128.1,127.8,60.7$, $56.8,51.4,50.0,40.0,30.9,22.7,20.8$. 
$\left(R^{*}, R^{*}\right)$ - Methyl [1-(1-phenylethyl)piperidin-2-yl]acetate (14). Following the general procedure for cyclization of mono protected amine, amine 11 (7.4 mmol, $2.20 \mathrm{~g}), \mathrm{K}_{2} \mathrm{CO}_{3}(1.30 \mathrm{~g})$ and $\mathrm{NaI}(1.04 \mathrm{~g})$ afforded, after purification on silica gel (ethyl acetate:cyclohexane 1:3), piperidine 14 as a yellow oil (1.35 g, 70\%): IR (film, $\mathrm{cm}^{-1}$ ) 2990, 1731, 1618, 1522, 1441, 1262; ${ }^{1} \mathrm{H}$ NMR (400 MHz, $\left.\mathrm{CDCl}_{3}\right) \delta$ 7.40-7.20 (5H, m), $3.90(1 \mathrm{H}, \mathrm{q}, \mathrm{J}=6.5 \mathrm{~Hz}), 3.70(3 \mathrm{H}, \mathrm{s}), 3.45$ $(2 \mathrm{H}, \mathrm{t}, \mathrm{J}=6.5 \mathrm{~Hz}), 2.75(1 \mathrm{H}, \mathrm{m}), 2.35(1 \mathrm{H}, \mathrm{dd}, \mathrm{J}=14.5$ and $6.0 \mathrm{~Hz}), 2.30(1 \mathrm{H}, \mathrm{dd}, \mathrm{J}=14.5$ and $5.5 \mathrm{~Hz}), 1.80-1.35(6 \mathrm{H}, \mathrm{m}), 1.30(3 \mathrm{H}, \mathrm{d}, \mathrm{J}=6.5 \mathrm{~Hz}) ;{ }^{13} \mathrm{C} \mathrm{NMR}\left(100 \mathrm{MHz}, \mathrm{CDCl}_{3}\right): \delta 172.6$, 143.2, 128.4, 127.1, 126.8, 55.4, 51.8, 51.5, 44.9, 38.1, 34.4, 32.3, 24.7, 23.4; HRMS Calcd. $\mathrm{C}_{16} \mathrm{H}_{23} \mathrm{NO}_{2}\left[\mathrm{M}^{+}\right]-\mathrm{CH}_{3}: 246.1494$. Found: 246.1469 .

\section{General procedure for preparation of Weinreb amides}

To a cold $\left(0^{\circ} \mathrm{C}\right)$ stirred solution of $N, O$-dimethylhydroxylamine hydrochloride (2 equiv.) in dry dichloromethane $(5 \mathrm{~mL} / \mathrm{mmol}$ of amine) a commercial $2.0 \mathrm{M}$ solution of trimethylaluminium in hexane ( 2 equiv.) was slowly added. The mixture was stirred at room temperature for 2 hours and amine (12 or 14) (1 equiv.) diluted in dichloromethane ( $2 \mathrm{~mL} / \mathrm{mmol}$ of pyrrolodine) was added. The resulting solution was stirred at room temperature until TLC indicated that the reaction had gone to completion. Then the mixture was carefully quenched with a saturated aqueous solution of $\mathrm{NH}_{4} \mathrm{Cl}$ and extracted with dichloromethane. The organic extract was dried, filtered and concentrated in vacuo. The crude Weinreb amide thus obtained was purified by column chromatography.

$\left(R^{*}, \quad R^{*}\right) \quad N$-methoxy- $N$-methyl-2-[1-(1-phenylethyl)pyrrolidin-2-yl]acetamide $\quad$ (15). Following the general procedure for preparation of Weinreb amides, pyrrolidine 12 (2.02 mmol, $0.50 \mathrm{~g})$ and $\mathrm{N}, \mathrm{O}$-dimethylhydroxylamine hydrochloride $(0.40 \mathrm{~g})$ afforded, after purification on silica gel (ethyl acetate:methanol 5:1), Weinreb amide 15 as a yellow oil (0.43 g, 78\%): IR (film, $\left.\mathrm{cm}^{-1}\right)$ 3042, 2964, 1656, 1605, 1531, 1452, 1258; ${ }^{1} \mathrm{H}$ NMR $\left(400 \mathrm{MHz}, \mathrm{CDCl}_{3}\right) \delta 7.75(2 \mathrm{H}, \mathrm{d}, \mathrm{J}=$ $8.0 \mathrm{~Hz}), 7.45(3 \mathrm{H}, \mathrm{m}), 4.15(1 \mathrm{H}, \mathrm{bs}), 3.80(3 \mathrm{H}, \mathrm{s}), 3.75(1 \mathrm{H}, \mathrm{m}), 3.40(1 \mathrm{H}, \mathrm{bs}), 3.30(1 \mathrm{H}, \mathrm{m})$, $3.10(3 \mathrm{H}, \mathrm{s}), 3.00(1 \mathrm{H}, \mathrm{bs}), 2.80(1 \mathrm{H}, \mathrm{m}), 2.00(2 \mathrm{H}, \mathrm{m}), 1.75(3 \mathrm{H}, \mathrm{d}, \mathrm{J}=6.5 \mathrm{~Hz}), 1.70(1 \mathrm{H}, \mathrm{m})$, $1.60(1 \mathrm{H}, \mathrm{m}) ;{ }^{13} \mathrm{C} \mathrm{NMR}\left(100 \mathrm{MHz}, \mathrm{CDCl}_{3}\right): \delta 170.9,135.8,129.2,129.1,128.3,61.6,61.5,60.3$, 49.5, 35.1, 32.0, 31.0, 22.3, 18.9; HRMS Calcd. $\mathrm{C}_{16} \mathrm{H}_{24} \mathrm{~N}_{2} \mathrm{O}_{2}\left[\mathrm{M}^{+}\right]$: 276.1834. Found: 276.1824.

$\left(R^{*}, R^{*}\right) \boldsymbol{N}$-methoxy- $\boldsymbol{N}$-methyl-2-[1-(1-phenylethyl)piperidin-2-yl]acetamide (16). Following the general procedure for preparation of Weinreb amides, piperidine 14 (1.91 mmol, $0.50 \mathrm{~g})$ and $\mathrm{N}, \mathrm{O}$-dimethylhydroxylamine hydrochloride $(0.38 \mathrm{~g})$ afforded, after purification on silica gel (ethyl acetate:methanol 5:1), Weinreb amide 16 as a yellow oil $(0.51 \mathrm{~g}, 92 \%)$ : IR (film, $\mathrm{cm}^{-1}$ ) 3037, 2955, 1655, 1612, 1542, 1454, 1245; ${ }^{1} \mathrm{H}$ NMR (400 MHz, $\left.\mathrm{CDCl}_{3}\right) \delta 7.75(2 \mathrm{H}, \mathrm{dd}, \mathrm{J}=8.0$ and $1.5 \mathrm{~Hz}), 7.45(3 \mathrm{H}, \mathrm{m}), 4.50(1 \mathrm{H}, \mathrm{q}, \mathrm{J}=7.0 \mathrm{~Hz}), 3.75(3 \mathrm{H}, \mathrm{s}), 3.45(3 \mathrm{H}, \mathrm{m}), 3.25(1 \mathrm{H}, \mathrm{dd}, \mathrm{J}=$ 18.0 and $6.5 \mathrm{~Hz}), 3.20(3 \mathrm{H}, \mathrm{s}), 2.95(1 \mathrm{H}, \mathrm{dd}, \mathrm{J}=18.0$ and $4.0 \mathrm{~Hz}), 1.90(3 \mathrm{H}, \mathrm{d}, \mathrm{J}=7.0 \mathrm{~Hz}), 1.90$ $1.30(6 \mathrm{H}, \mathrm{m}) ;{ }^{13} \mathrm{C}$ NMR $\left(100 \mathrm{MHz}, \mathrm{CDCl}_{3}\right): \delta 171.3,129.2$, 128.1, 127.3, 61.5, 57.6, 53.2, 44.5, 33.6, 32.1, 31.9, 31.6, 22.9, 21.1.

$\left(R *, R^{*}\right)$ 1-[1-(1-phenylethyl)pyrrolidin-2-yl]propan-2-one) (17). A commercial 3M solution of methyl magnesium bromide (3 equiv., $7.60 \mathrm{mmol}, 2.53 \mathrm{~mL}$ ) in diethyl ether was added to a solution of Weinreb amide 15 (1 equiv., $2.53 \mathrm{mmol}, 0.70 \mathrm{~g})$ in $\mathrm{THF}(25 \mathrm{~mL})$ at $0^{\circ} \mathrm{C}$. The 
mixture was stirred at $0^{\circ} \mathrm{C}$ for 30 minutes, and then a saturated aqueous solution of ammonium chloride was added. The mixture was then extracted with diethyl ether. Combined organic extracts were dried, filtered and evaporated. The crude ketone such obtained was purified by column chromatography on neutral aluminium oxide (ethyl acetate:cyclohexane 1:3) to afford $0.40 \mathrm{~g}$ of 17 (68\% yield) as a pale yellow oil: IR (film, $\mathrm{cm}^{-1}$ ) 3012, 2970, 1709, 1610, 1525, 1448, 1236; ${ }^{1} \mathrm{H}$ NMR (400 MHz, $\left.\mathrm{CDCl}_{3}\right) \delta 7.20(5 \mathrm{H}, \mathrm{m}), 3.70(1 \mathrm{H}, \mathrm{q}, \mathrm{J}=6.5 \mathrm{~Hz}), 3.10(1 \mathrm{H}, \mathrm{m})$, $2.70(2 \mathrm{H}, \mathrm{m}), 2.35(2 \mathrm{H}, \mathrm{m}), 2.00(3 \mathrm{H}, \mathrm{s}), 1.80(1 \mathrm{H}, \mathrm{m}), 1.60(2 \mathrm{H}, \mathrm{m}), 1.40(3 \mathrm{H}, \mathrm{d}, \mathrm{J}=6.5 \mathrm{~Hz})$, $1.35(1 \mathrm{H}, \mathrm{m}) ;{ }^{13} \mathrm{C} \mathrm{NMR}\left(100 \mathrm{MHz}, \mathrm{CDCl}_{3}\right): \delta 208.7,143.0,128.3,127.8,127.0,60.7,56.0,49.8$, 49.0, 30.9, 30.4, 23.1, 19.2; HRMS Calcd. $\mathrm{C}_{15} \mathrm{H}_{21} \mathrm{NO}\left[\mathrm{M}^{+}\right]$: 231.1623 . Found: 231.1617.

$\left(R^{*}, \quad R^{*}\right)$ 2-[(2-methyl-1,3-dioxolan-2-yl)methyl]-1-(1-phenylethyl)pyrrolidine (18). A solution of ketone 17 ( 1 equiv., $1.08 \mathrm{mmol}, 0.25 \mathrm{~g}$ ), trimethylorthoformate (5 equiv., $5.40 \mathrm{mmol}$, $0.60 \mathrm{~g})$, ethylene glycol (5 equiv., $5.40 \mathrm{mmol}, 0.33 \mathrm{~g})$ ) and para-toluene sulfonic acid (0.04 equiv., $0.04 \mathrm{mmol}, 0.008 \mathrm{~g}$ ) was stirred at room temperature until TLC showed no starting ketone. The mixture was then diluted with dichloromethane and washed with a saturated aqueous solution of sodium hydrogencarbonate. The organic layer was dried over sodium sulfate, filtered over celite ${ }^{\circledR}$ and concentrated in vacuo to afford the crude protected adduct. A purification by column chromatography (ethyl acetate:cyclohexane 1:3) afforded $0.22 \mathrm{~g}$ of $\mathbf{1 8}$ (74\% yield) as a pale yellow oil: IR (film, $\mathrm{cm}^{-1}$ ) 3015, 2951, 1609, 1548, 1353, 1255; ${ }^{1} \mathrm{H}$ NMR (400 MHz, $\left.\mathrm{CDCl}_{3}\right) \delta 7.20(5 \mathrm{H}, \mathrm{m}), 3.85(4 \mathrm{H}, \mathrm{m}), 3.70(1 \mathrm{H}, \mathrm{q}, \mathrm{J}=6.5 \mathrm{~Hz}), 2.90(1 \mathrm{H}, \mathrm{m}), 2.65(1 \mathrm{H}, \mathrm{m}), 2.30$ $(1 \mathrm{H}, \mathrm{q}, \mathrm{J}=7.0 \mathrm{~Hz}), 2.10(1 \mathrm{H}, \mathrm{dd}, \mathrm{J}=14.5$ and $2.0 \mathrm{~Hz}), 1.80-1.50(5 \mathrm{H}, \mathrm{m}), 1.30(3 \mathrm{H}, \mathrm{d}, \mathrm{J}=6.5$ $\mathrm{Hz}), 1.10(3 \mathrm{H}, \mathrm{s}) ;{ }^{13} \mathrm{C} \mathrm{NMR}\left(100 \mathrm{MHz}, \mathrm{CDCl}_{3}\right): \delta 143.6,129.0,128.3,128.1,111.2,64.5,64.2$, $59.8,48.3,31.6,27.3,26.8,24.6,22.5,18.3$.

2-[(2-methyl-1,3-dioxolan-2-yl)methyl]pyrrolidine (1). To a stirred solution of aminoketal 18 ( 1 equiv., $1.09 \mathrm{mmol}, 0.30 \mathrm{~g})$ in anhydrous methanol $(10 \mathrm{~mL})$ was added $10 \% \mathrm{Pd} / \mathrm{C}(60 \mathrm{mg})$ then ammonium formate ( 5 equiv., $5.84 \mathrm{mmol}, 0.38 \mathrm{~g}$ ). The resulting suspension was heated at reflux for 5 hours. After cooling at room temperature, the catalyst was removed by filtration on celite ${ }^{\circledR}$. The residue obtained after evaporation of the solvent was diluted with dichloromethane. This organic phase was washed with a saturated aqueous solution of sodium hydrogencarbonate. The aqueous phase was extracted with dichloromethane and the combined organic extracts were dried, filtered then concentrated to afford $0.13 \mathrm{~g}$ of pure $\beta$-aminoketal 1 (73\% yield): IR (film, $\mathrm{cm}^{-1}$ ) 3618, 2961, 1356, 1262; ${ }^{1} \mathrm{H}$ NMR (400 MHz, $\left.\mathrm{CDCl}_{3}\right) \delta 3.90$ (4H, m), 3.10 (1H, m), 2.95 $(1 \mathrm{H}, \mathrm{m}), 2.70(1 \mathrm{H}, \mathrm{m}), 2.00(1 \mathrm{H}, \mathrm{bs}), 1.80(1 \mathrm{H}, \mathrm{m}), 1.75(2 \mathrm{H}, \mathrm{dd}, \mathrm{J}=10$ and $5 \mathrm{~Hz}), 1.60(2 \mathrm{H}, \mathrm{m})$, $1.30(3 \mathrm{H}, \mathrm{s}), 1.20(1 \mathrm{H}, \mathrm{m}) ;{ }^{13} \mathrm{C} \mathrm{NMR}\left(100 \mathrm{MHz}, \mathrm{CDCl}_{3}\right): \delta 109.6,64.6,64.4,54.9,45.9,44.4$, $32.3,24.4,24.2$.

$\left(S^{*}, S^{*}\right)$ 5,5-(1,3-dioxolan-2yl)-7-[(1E)-hex-1-en-1yl)]indolizidine (20). To a stirred solution of trans-hepten-2-al (1.2 equiv., $1.05 \mathrm{mmol}, 0.12 \mathrm{~g})$ in dichloromethane $(10 \mathrm{ml})$ was added $\mathrm{MgSO}_{4}$ (ca $1 \mathrm{~g}$ ) followed by a solution of $\beta$-aminoketal 1 (1.0 equiv., $0.88 \mathrm{mmol}, 0.15 \mathrm{~g})$ in dichloromethane $(5 \mathrm{~mL})$. The resulting solution was heated at reflux until complete disappearance (TLC monitoring) of the amine $(3 \mathrm{~h})$ then cooled to room temperature and transferred via a cannula to a solution of dry para-toluenesulfonic acid (1 equiv., $0.88 \mathrm{mmol}$, 
$0.17 \mathrm{~g})$ in toluene $(15 \mathrm{~mL})$. The resulting mixture was heated at $70^{\circ} \mathrm{C}$ for 12 hours. After being cooled to room temperature, saturated aqueous $\mathrm{NaHCO}_{3}(15 \mathrm{~mL})$ was added and the mixture was extracted with ethyl acetate. The combined organic extracts were dried, filtered and evaporated. The residue was purified by column chromatography to give $0.09 \mathrm{~g}$ of indolizidine $\mathbf{2 0}$ (40\% yield) ): IR (film, cm ${ }^{-1}$ ) 2956, 1550, 1377; ${ }^{1} \mathrm{H}$ NMR (400 MHz, $\left.\mathrm{CDCl}_{3}\right) \delta 5.45(1 \mathrm{H}, \mathrm{dt}, \mathrm{J}=15.5$ and $7.0 \mathrm{~Hz}), 5.25(1 \mathrm{H}, \mathrm{dd}, \mathrm{J}=15.5$ and $7.5 \mathrm{~Hz}), 3.80(4 \mathrm{H}, \mathrm{s}), 2.95(1 \mathrm{H}, \mathrm{t}, \mathrm{J}=9.0 \mathrm{~Hz}), 2.50(1 \mathrm{H}$, m), $2.05(1 \mathrm{H}, \mathrm{m}), 1.85(3 \mathrm{H}, \mathrm{m}), 1.80-1.50(5 \mathrm{H}, \mathrm{m}), 1.40-1.10(6 \mathrm{H}, \mathrm{m}), 0.80(1 \mathrm{H}, \mathrm{m}), 0.75(3 \mathrm{H}, \mathrm{t}$, $\mathrm{J}=7.0 \mathrm{~Hz}) ;{ }^{13} \mathrm{C}$ NMR $\left(100 \mathrm{MHz}, \mathrm{CDCl}_{3}\right): \delta 132.3,131.9,108.3,64.3,64.2,63.2,61.6,51.6$, 41.6, 40.1, 31.8, 31.3, 30.1, 22.1, 21.0, 13.9.

$(S *, S *)$ 5,5-(1,3-dithian-2yl)-7-[(1E)-hex-1-en-1yl)]indolizidine (21). To a stirred solution of indolizidine ketal 20 (1 equiv., $0.19 \mathrm{mmol}, 0.05 \mathrm{~g}$ ) in dichloromethane (10 mL) was added dropwise, at room temperature, ethanedithiol (5 equiv., $0.95 \mathrm{mmol}, 0.08 \mathrm{~mL}$ ) then boron trifluoride etherate (5 equiv., $0.95 \mathrm{mmol}, 0.13 \mathrm{~mL}$ ). After $1 \mathrm{~h}$ of stirring, an excess of $1 \mathrm{M}$ aqueous $\mathrm{NaOH}$ was added and the resulting mixture was extracted with dichloromethane. The combined organic extracts were dried, filtered and evaporated. The residue, purified by column chromatography (ethyl acetate:cyclohexane 2:1), gave $0.045 \mathrm{~g}$ of the corresponding dithiolane derivative 21 (79\% yield): IR (film, cm ${ }^{-1}$ ) 2923, 2870, 1638, 1458, 1355; ${ }^{1} \mathrm{H} \mathrm{NMR} \mathrm{(400} \mathrm{MHz,}$ $\left.\mathrm{CDCl}_{3}\right) \delta 5.60(1 \mathrm{H}, \mathrm{dt}, \mathrm{J}=15.0$ and $6.5 \mathrm{~Hz}), 5.35(1 \mathrm{H}, \mathrm{dd}, \mathrm{J}=15.0$ and $9.0 \mathrm{~Hz}), 3.05(1 \mathrm{H}, \mathrm{t}, \mathrm{J}=$ $9.5 \mathrm{~Hz}), 2.95(\mathrm{dt}, \mathrm{J}=9.0$ and $4.5 \mathrm{~Hz}), 2.70-2.50(5 \mathrm{H}, \mathrm{m}), 2.10(1 \mathrm{H}, \mathrm{m}), 2.00(1 \mathrm{H}, \mathrm{m}), 1.90-1.60$ $(5 \mathrm{H}, \mathrm{m}), 1.40-1.20(6 \mathrm{H}, \mathrm{m}), 0.90(1 \mathrm{H}, \mathrm{m}), 0.80(3 \mathrm{H}, \mathrm{t}, \mathrm{J}=7.0 \mathrm{~Hz}) ;{ }^{13} \mathrm{C} \mathrm{NMR}\left(100 \mathrm{MHz}, \mathrm{CDCl}_{3}\right)$ : $\delta$ 132.6, 131.9, 62.1, 59.9, 59.0, 51.7, 44.6, 42.1, 31.8, 31.3, 29.9, 22.6, 22.4, 22.1, 20.5, 14.1; HRMS Calcd. $\mathrm{C}_{14} \mathrm{H}_{27} \mathrm{~N}\left[\mathrm{M}^{+}\right]$: 207.3582. Found: 207.3558.

\section{( \pm )-Indolizidine 209D (22)}

To a stirred solution of thioketal 21 (1 equiv., $0.13 \mathrm{mmol}, 0.040 \mathrm{~g})$ in absolute ethanol (10 $\mathrm{mL})$ was added freshly prepared W2 Raney-nickel ${ }^{16}(100 \mathrm{mg})$. The resulting suspension was heated at reflux for $30 \mathrm{~min}$., cooled to room temperature before filtration over celite ${ }^{\circledR}$ then concentration of the filtrate under reduced pressure. The residue was dissolved in $1 \mathrm{M} \mathrm{NaOH}$ and the indolizidine was extracted with dichloromethane. The combined organic extracts were dried, filtered and evaporated to give $0.022 \mathrm{~g}$ of $\left( \pm\right.$ )-indolizidine 209D 22 (83\% yield): IR (film, $\mathrm{cm}^{-1}$ ) 2931, 2863, 1455; ${ }^{1} \mathrm{H}$ NMR (400 MHz, $\left.\mathrm{CDCl}_{3}\right) \delta 3.25(1 \mathrm{H}, \mathrm{m}), 2.00-1.60(10 \mathrm{H}, \mathrm{m}), 1.55-1.10(13 \mathrm{H}, \mathrm{m})$, $0.85(3 \mathrm{H}, \mathrm{t}, \mathrm{J}=7.0 \mathrm{~Hz}) ;{ }^{13} \mathrm{C} \mathrm{NMR}\left(100 \mathrm{MHz}, \mathrm{CDCl}_{3}\right): \delta 65.1,64.0,51.3,34.3,31.8,31.1,30.8$, 30.5, 29.5, 25.7, 24.7, 22.5, 20.2, 14.2; HRMS Calcd. $\mathrm{C}_{14} \mathrm{H}_{27} \mathrm{~N}\left[\mathrm{M}^{+}\right]: 209.3740$. Found: 209.3729.

\section{Benzyl 2-\{2-[methoxy(methyl)amino]-2-oxoethyl\}piperidine-1-carboxylate (25)}

To a stirred solution of amide 16 ( 1 equiv., $1.37 \mathrm{mmol}, 0.4 \mathrm{~g})$ in anhydrous methanol (20 $\mathrm{mL})$ was added $10 \% \mathrm{Pd} / \mathrm{C}(50 \mathrm{mg}$ ) then ammonium formate (5 equiv., $6.85 \mathrm{mmol}, 0.44 \mathrm{~g})$. The resulting suspension was heated at reflux for 5 hours. After cooling at room temperature, the catalyst was removed by filtration on celite ${ }^{\circledR}$. The residue obtained after evaporation of the solvent was diluted with dichloromethane $(15 \mathrm{~mL})$ and a $0.4 \mathrm{M}$ aqueous sodium carbonate (2 equiv., $2.74 \mathrm{mmol}, 6.9 \mathrm{~mL}$ ). The mixture was cooled to $0^{\circ} \mathrm{C}$ before addition of 
benzylchloroformate ( 2 equiv., $2.74 \mathrm{mmol}, 0.39 \mathrm{~mL}$ ). The ice bath was removed and the solution stirred at room temperature. After three hours the solution was diluted with dichloromethane and the combined organic material was washed with water, dried over anhydrous sodium sulfate, filtered and concentrated. The crude $N$-protected piperidine thus obtained was purified by column chromatography over neutral aluminium oxide (ethyl acetate) to afford $0.31 \mathrm{~g}$ of piperidine 25 (70\% yield): IR (film, $\mathrm{cm}^{-1}$ ) 3010, 2953, 1672, 1664, 1365; ${ }^{1} \mathrm{H}$ NMR (400 MHz, $\left.\mathrm{CDCl}_{3}\right) \delta 7.20(5 \mathrm{H}, \mathrm{m}), 5.00(2 \mathrm{H}, \mathrm{s}), 4.70(1 \mathrm{H}, \mathrm{m}), 4.00(1 \mathrm{H}, \mathrm{d}, \mathrm{J}=11.7 \mathrm{~Hz}), 3.50(3 \mathrm{H}, \mathrm{s}), 3.00$ $(3 \mathrm{H}, \mathrm{s}), 2.80(1 \mathrm{H}, \mathrm{t}, \mathrm{J}=11.7 \mathrm{~Hz}), 2.60(2 \mathrm{H}, \mathrm{m}), 1.70-1.30(6 \mathrm{H}, \mathrm{m}) ;{ }^{13} \mathrm{C} \mathrm{NMR}(100 \mathrm{MHz}$, $\left.\mathrm{CDCl}_{3}\right): \delta 171.5,155.3,137.0,136.8,129.1,128.4,128.0,127.8,127.5,127.2,67.0,61.3,48.0$, $39.8,32.9,32.1,28.3,25.3,18.9$.

\section{Benzyl 2-(2-oxopropyl)piperidine-1-carboxylate (26)}

A commercial 3M solution of methyl magnesium bromide (3 equiv., $1.87 \mathrm{mmol}, 0.6 \mathrm{~mL}$ ) in diethyl ether was added to a solution of Weinreb amide 25 ( 1 equiv., $0.63 \mathrm{mmol}, 0.20 \mathrm{~g}$ ) in THF $(15 \mathrm{~mL})$ at $0^{\circ} \mathrm{C}$. The mixture was stirred at $0^{\circ} \mathrm{C}$ for 30 minutes, and then a saturated $\mathrm{NH}_{4} \mathrm{Cl}$ aqueous solution was added. The mixture was then extracted with diethyl ether. The combined organic extracts were dried, filtered and evaporated. The crude ketone thus obtained was purified by column chromatography on neutral aluminium oxide (ethyl acetate:cyclohexane 1:3) to afford $0.12 \mathrm{~g}$ of 26 (67\% yield) as a pale yellow oil: IR (film, $\left.\mathrm{cm}^{-1}\right)$ 3019, 2965, 1700, 1684, 1531, 1452, 1253; ${ }^{1} \mathrm{H}$ NMR (400 MHz, $\left.\mathrm{CDCl}_{3}\right) \delta 7.30(5 \mathrm{H}, \mathrm{m}), 5.10(1 \mathrm{H}, \mathrm{d}, \mathrm{J}=12.4 \mathrm{~Hz}), 5.00(1 \mathrm{H}, \mathrm{d}$, $\mathrm{J}=12.4 \mathrm{~Hz}), 4.80(1 \mathrm{H}, \mathrm{m}), 4.10(1 \mathrm{H}, \mathrm{d}, \mathrm{J}=10.9 \mathrm{~Hz}), 2.80(1 \mathrm{H}, \mathrm{t}, \mathrm{J}=10.9 \mathrm{~Hz}), 2.60(2 \mathrm{H}, \mathrm{d}, \mathrm{J}=$ $7.9 \mathrm{~Hz}), 2.10(3 \mathrm{H}, \mathrm{s}), 1.70-1.30(6 \mathrm{H}, \mathrm{m}) ;{ }^{13} \mathrm{C} \mathrm{NMR}\left(100 \mathrm{MHz}, \mathrm{CDCl}_{3}\right): \delta 209.1,155.3,136.8$, $128.5,128.0,127.8,67.1,47.5,44.3,39.8,30.0,28.3,25.2,18.8$.

\section{$\left(R^{*}, S^{*}\right)$ and $\left(R^{*}, R^{*}\right)$-benzyl 2-(2-hydroxypropyl)piperidine-1-carboxylate 27 and 28}

A commercial 1M solution of Superhydride ${ }^{\circledR}$ (1.1 equiv., $\left.0.32 \mathrm{mmol}, 0.32 \mathrm{~mL}\right)$ in dichloromethane was added to a solution of ketone 26 (1 equiv., $0.29 \mathrm{mmol}, 0.08 \mathrm{~g}$ ) in anhydrous dichloromethane $(10 \mathrm{~mL})$ at $-78^{\circ} \mathrm{C}$. The mixture was stirred at $-78^{\circ} \mathrm{C}$ until $\mathrm{TLC}$ showed no starting ketone. Methanol $(5 \mathrm{~mL})$ was added then a saturated $\mathrm{NH}_{4} \mathrm{Cl}$ aqueous solution $(5 \mathrm{~mL})$. The mixture was warmed to room temperature before the addition of water $(20 \mathrm{~mL})$. The resulting solution was extracted with diethyl ether, the combined organic extracts were dried over sodium sulfate, filtered and concentrated in vacuo to afford a mixture of two diastereoisomeric alcohols $\mathbf{2 7}$ and $\mathbf{2 8}$ in a 7:3 ratio. Purification by column chromatography (ethyl acetate:cyclohexane 2:1) afforded $0.05 \mathrm{~g}$ of $\mathbf{2 7}$ and $0.02 \mathrm{~g}$ of $\mathbf{2 8}$ (90\% overall yield).

\section{Benzyl $\left(2 R^{*}\right)$-2-[(2S*)-2-hydroxypropyl]piperidine-1-carboxylate (27)}

IR (film, $\left.\mathrm{cm}^{-1}\right)$ 3446, 2934, 1684, 1551, 1343, 1262; ${ }^{1} \mathrm{H}$ NMR (400 MHz, $\left.\mathrm{CDCl}_{3}\right) \delta 7.40(5 \mathrm{H}$, m), $5.10(1 \mathrm{H}, \mathrm{d}, \mathrm{J}=12.5 \mathrm{~Hz}), 5.00(1 \mathrm{H}, \mathrm{d}, \mathrm{J}=12.5 \mathrm{~Hz}), 4.55(1 \mathrm{H}, \mathrm{bs}), 4.40(1 \mathrm{H}, \mathrm{d}, \mathrm{J}=5.5 \mathrm{~Hz})$, $4.05(1 \mathrm{H}, \mathrm{d}, \mathrm{J}=11.9 \mathrm{~Hz}), 3.70(1 \mathrm{H}, \mathrm{m}), 2.90(1 \mathrm{H}, \mathrm{t}, \mathrm{J}=11.9 \mathrm{~Hz}), 1.80-1.20(8 \mathrm{H}, \mathrm{m}), 1.30(3 \mathrm{H}$, $\mathrm{d}, \mathrm{J}=6.5 \mathrm{~Hz}) ;{ }^{13} \mathrm{C} \mathrm{NMR}\left(100 \mathrm{MHz}, \mathrm{CDCl}_{3}\right): \delta 157.0,136.7,128.5,128.0,127.9,67.2,66.4$, 49.0, 39.6, 39.4, 28.5, 25.4, 22.9, 19.0; HRMS Calcd. $\mathrm{C}_{16} \mathrm{H}_{23} \mathrm{NO}_{3}\left[\mathrm{M}^{+}\right]$: 277.3626. Found: 277.3611. 
Benzyl (2R*)-2-[(2R*)-2-hydroxypropyl]piperidine-1-carboxylate (28). IR (film, $\mathrm{cm}^{-1}$ ) 3440, 2943, 1664, 1545, 1257; ${ }^{1} \mathrm{H}$ NMR (400 MHz, $\left.\mathrm{CDCl}_{3}\right) \delta 7.30(5 \mathrm{H}, \mathrm{m}), 5.10(1 \mathrm{H}, \mathrm{d}, \mathrm{J}=12.0 \mathrm{~Hz})$, $5.05(1 \mathrm{H}, \mathrm{d}, \mathrm{J}=12.0 \mathrm{~Hz}), 4.45(1 \mathrm{H}, \mathrm{d}, \mathrm{J}=7.8 \mathrm{~Hz}), 4.10(1 \mathrm{H}, \mathrm{bs}), 4.05(1 \mathrm{H}, \mathrm{d}, \mathrm{J}=13.8 \mathrm{~Hz}), 3.45$ $(1 \mathrm{H}, \mathrm{m}), 2.70(1 \mathrm{H}, \mathrm{td}, \mathrm{J}=12.8$ and $2.5 \mathrm{~Hz}), 1.95(1 \mathrm{H}, \mathrm{td}, \mathrm{J}=7.0$ and $2.0 \mathrm{~Hz}), 1.70-1.20(7 \mathrm{H}, \mathrm{m})$, $1.10(3 \mathrm{H}, \mathrm{d}, \mathrm{J}=6.0 \mathrm{~Hz}) ;{ }^{13} \mathrm{C} \mathrm{NMR}\left(100 \mathrm{MHz}, \mathrm{CDCl}_{3}\right): \delta 155.0,136.0,128.5,128.1,127.9,67.5$, $63.3,47.4,39.4,29.7,29.3,25.5,22.5,19.1$.

\section{( \pm )-Allosedridine (29)}

A suspension of $N$-protected piperidine 27 (1 equiv., $0.18 \mathrm{mmol}, 0.05 \mathrm{~g})$ and $\mathrm{Pd}(\mathrm{OH})_{2}(10 \mathrm{mg})$ in $\mathrm{MeOH}(5 \mathrm{~mL})$, under an hydrogen atmosphere, was stirred for $24 \mathrm{~h}$. After cooling to room temperature, the catalyst was removed by filtration on celite ${ }^{\circledR}$. The filtrate was evaporated to give $0.24 \mathrm{~g}$ of $\left( \pm\right.$ )-allosedridine 29 (95\% yield): IR (film, $\mathrm{cm}^{-1}$ ) 3685, 3679, 2932, 1661, 1436, 1357; ${ }^{1} \mathrm{H}$ NMR $\left(400 \mathrm{MHz}, \mathrm{CDCl}_{3}\right) \delta 4.01(1 \mathrm{H}, \mathrm{dq}, \mathrm{J}=11.5$ and $6.5 \mathrm{~Hz}), 3.45(2 \mathrm{H}, \mathrm{m}), 3.05(1 \mathrm{H}, \mathrm{d}, \mathrm{J}=$ $13.5 \mathrm{~Hz}), 2.65(1 \mathrm{H}, \mathrm{t}, \mathrm{J}=10.5 \mathrm{~Hz}), 2.50(\mathrm{td}, \mathrm{J}=14.0$ and $3.0 \mathrm{~Hz}), 1.75(1 \mathrm{H}, \mathrm{m}), 1.60(2 \mathrm{H}, \mathrm{m})$, $1.45(2 \mathrm{H}, \mathrm{m}), 1.35-1.15(2 \mathrm{H}, \mathrm{m}), 1.15(3 \mathrm{H}, \mathrm{d}, \mathrm{J}=6.5 \mathrm{~Hz}), 1.07(1 \mathrm{H}, \mathrm{m}) ;{ }^{13} \mathrm{C} \mathrm{NMR}(100 \mathrm{MHz}$, $\left.\mathrm{CDCl}_{3}\right): \delta 70.5,57.8,46.5,43.8,35.0,26.8,24.7,23.9 ;$ HRMS Calcd. $\mathrm{C}_{8} \mathrm{H}_{17} \mathrm{~N}\left[\mathrm{M}^{+}\right]$: 127.2290. Found: 127.2275 .

\section{References}

1. Schneider, M. J. Alkaloids: Chemical and Biological Perspectives, Pelletier, S. W., Eds. Pergamon 1996, Vol.10, Chapter 2, p155.

2. (a) Bailey, P. D.; Millwood, P. A.; Smith, P. D. J. Chem. Soc., Chem. Commun. 1998, 633. (b) Weintraub, P. M.; Sabol, J. S.; Kane, J. M.; Borcherding, D. R. Tetrahedron, 2003, 59, 2953. (c) Buffat, M. G. P. Tetrahedron, 2004, 60, 1701.

3. (a) Ciblat, S., Besse, P., Papastergiou, V., Veschambre, H., Canet, J.-L., Troin, Y. Tetrahedron: Asymmetry 2000, 11, 2221. (b) Ciblat, S., Calinaud, P., Canet, J.-L., Troin, Y. J. Chem. Soc., Perkin Trans.1 2000, 353. (c) Rougnon-Glasson, S.; Tratrat, C.; Canet, J.-L.; Chalard, P.; Troin, Y. Tetrahedron: Asymmetry 2004, 15, 1561.

4. Bariau, A.; Canet, J.-L.; Chalard, P.; Troin Y. Tetrahedron: Asymmetry 2005, 16, 3650.

5. Davies, S. G.; Ichihara, O. Tetrahedron: Asymmetry 1991, 2, 183.

6. O'Brien, P.; Porter, D. W.; Smith, N. M. Synlett 2000, 1336.

7. Witiak, D. T.; Tomita, K.; Patch, R. J.; Enna, S. J. J. Med. Chem. 1981, 24, 788.

8. Enders, D.; Wiedemann. J. Liebigs Annalen / Recueil 1997, 4, 699.

9. Hashimoto, N.; Funatomi, T.; Misaki, T.; Tanabe, Y. Tetrahedron 2006, 62, 2214.

10. Pilli, R. A.; Dias, L. C.; Maldaner, A. O. J. Org. Chem. 1995, 60, 717.

11. (a) Levin, J. I.; Turos, E.; Weinreb, S. M. Synth. Commun. 1982, 12, 989. (b) Sibi, M. P. Org. Proc. Prep. Int., 1993, 25, 15.

12. (a) Michael, J. P. J. Nat. Prod. Rep. 1997, 14, 619; (b) Kim, G; Jung, S.; Kim, W. J. Org. Lett. 2001, 3, 2985. (c) Kim, G.; Lee, E.-J. Tetrahedron: Asymmetry 2001, 12, 2073. (d) 
Back, T. G.; Nakajima, K. J. Org. Chem. 2000, 65, 4543. (e) Chenevert, R.; Zianari, G. M.; Morin, M. P.; Dasser, M. Tetrahedron: Asymmetry 1999, 10, 3117. (f) Takahat, H.; Kubota, M.; Ihara, K.; Okamoto, N.; Momose, T.; Azer, N.; Eldefrawi, A. T.; Eldefrawi, M. E. Tetrahedron: Asymmetry 1998, 9, 3289.

13. (a) Aronstam, R. S.; Daly, J. W.; Spandle, T. L.; Narayanan, T. K.; Albuquerque, E. X. Neurochem. Res., 1986, 11, 1227. (b) Daly, J. W.; Nishizawa, Y.; Padgett, W.; Tokuyama, T.; McCloskey, P. J.; Waykole, L.; Schultz, G. A.; Aronstam, R. S. Neurochem. Res. 1991, $16,1207$.

14. Peters, O.; Debaerdemaeker, T.; Friedrichsen, W. J. Chem. Soc., Perkin Trans. 1, 1999, 59.

15. Lopez, I.; Diez, A.; Rubiralta, M., Tetrahedron, 1996, 52, 8581.

16. (a) Mozingo, R. Org. Synth. 1941, 21, 15. (b) Augustine, R. L. In Catalytic Hydrogenation; M. Decker Inc., Ed.: New York, 1965, p23.

17. (a) Bisai, A.; Singh, V. K. Tetrahedron Lett. 2007, 48, 1907. (b) Davis, F. A.; Prasad, K. R.; Brad Nolt, M.; Wu, Y. Org. Lett., 2003, 5, 925. (c) Takahata, H.; Kubota, M.; Ikota, N. J. Org. Chem., 1999, 64, 8594.

18. (a) Louis, C.; Hootele, C. Tetrahedron: Asymmetry 1995, 6, 2149. (b) Littler, B. J.; Gallagher, T.; Boddy, I. K.; Riordan, P. Synlett 1997, 22. (c) Louis, C.; Hootele, C. Tetrahedron: Asymmetry 1997, 8, 109. (d) Fodor, G.; Brutuile, D.; Huber-Saunderson, C.; Letourneau, F. Tetrahedron, 1971, 27, 2055.

19. Munchhof, M. J.; Meyers, A. I. J. Am. Chem. Soc., 1995, 117, 5399. 